\title{
Terminologie de l'environnement et Sémantique des cadres
}

\author{
L'Homme, Marie-Claude \\ Observatoire de linguistique Sens-Texte (OLST) \\ Département de linguistique et de traduction \\ Université de Montreal \\ C.P. 6128 , succ. Centre-ville \\ Montréal (Québec), Canada, H3C 1J7 \\ mc.lhomme@umontreal.ca
}

\begin{abstract}
Résumé. Dans le présent article, nous montrons de quelle manière la Sémantique des cadres (Fillmore 1976 ; Fillmore \& Atkins 1982; Fillmore \& Baker 2010) et la méthodologie développée dans le projet FrameNet (Fillmore et al. 2003 ; Ruppenhofer et al. 2010) permettent de rendre compte du lexique spécialisé de l'environnement. Le travail se focalise sur des termes de nature prédicative : verbes (contaminer, menacer, reboiser), noms prédicatifs (agriculture, impact, recyclage) et adjectifs (génétiquement modifié, propre, protégé) En premier lieu, nous évoquons les hypothèses à l'origine de ce travail et décrivons les ressources que nous élaborons. Dans les ressources, trois niveaux de description sont interreliés : une ressource lexicale, des annotations contextuelles et un module de définition de cadres sémantiques. En second lieu, nous décrivons une partie des étapes méthodologiques menant à l'élaboration de ces ressources. La méthodologie est ascendante et s'appuie largement sur le contenu de corpus spécialisés.
\end{abstract}

\begin{abstract}
This article shows how Frame Semantics (Fillmore 1976; Fillmore \& Atkins 1982; Fillmore \& Baker 2010) and the methodology developed within the FrameNet project (Fillmore et al. 2003; Ruppenhofer et al. 2010) is used to account for specialized terms in the field of the environment. This works focuses on predicative units: verbs (contaminate, reforest, threaten), predicative nouns (agriculture, impact, recycling) and adjectives (clean, endangered, protected) First, the hypotheses that triggered this kind of analysis are discussed and the resource that we compile are presented. In this resource, three different descriptive modules are interconnected: a lexical module, a contextual annotations module, and a module that defines semantic frames. Secondly, a brief account of the methodological steps taken to compile these resources is given. The methodology is bottom-up and is heavily based on the content of specialized corpora.
\end{abstract}

\section{Introduction}

Les ressources terminologiques de format électronique se présentent grosso modo sous trois formes: banques de terminologie (par exemple, IATE 2015); thésaurus (ex. EIONET 2015); ontologies de domaine (ex. Envo 2015). Ces ressources visent le plus souvent à fournir des explications sur le lien entre les termes et les connaissances qu'ils expriment. Un accent particulier est mis sur la formulation de définitions consensuelles et la situation des concepts dénotés par les termes dans une structure dont l'épine dorsale est une taxinomie. Cette optique a comme conséquence de laisser peu de place aux renseignements de nature linguistique. L'utilisateur à la recherche d'information sur le fonctionnement linguistique des termes pourra se tourner vers des ressources générales, mais il est probable que celles-ci ne renferment qu'une partie des items lexicaux qui l'intéressent. De plus, les ressources générales n’ont pas comme objectif de donner un portrait de la manière dont les connaissances sont organisées dans les domaines de spécialité ${ }^{1}$. 
Afin de combler ces lacunes, nous proposons un modèle de description des termes qui tient compte de leurs propriétés lexico-sémantiques (leur structure argumentale, leur place dans le réseau lexical d'une langue, etc.) et qui relie cette description à une représentation conceptuelle. Le modèle s'inspire de la Sémantique des cadres (Fillmore 1976; Fillmore \& Atkins 1982; Fillmore \& Baker 2010) et la méthodologie développée dans le projet FrameNet (Fillmore et al. 2003 ; Ruppenhofer et al. 2010).

Dans le présent article, nous montrons de quelle manière ce modèle descriptif permet de rendre compte du lexique spécialisé de l'environnement. Nous évoquons les hypothèses à l'origine de ce travail, décrivons les ressources que nous élaborons et passons en revue les étapes méthodologiques que nous appliquons. Le travail décrit dans les pages qui suivent est en cours en ce sens que les termes du domaine n'ont pas encore tous été décrits, mais il est suffisamment avancé pour montrer qu'il est possible de coupler une description linguistique à une représentation des concepts du domaine.

\section{Le lexique de l'environnement et son traitement dans les ressources}

Le domaine de l'environnement se caractérise par la variété des connaissances auxquelles il fait appel, connaisances relevant de domaines scientifiques, techniques, sociaux et économiques. Les termes peuvent dénoter des éléments et composés chimiques (dioxyde de carbone, méthane), des ressources naturelles (bois, eau, pétrole), des dispositifs techniques (éolienne, turbine), des acteurs (agriculteur, environnementaliste), des lieux (atmosphère, cryosphère, océan), mais également des propriétés (potable, toxique, vert), des états (climat, condition, temps), des faits (amincissement, éroder, recul) et des activités (déversement, pollution, recyclage). Dans certains cas, ces connaissances ne sont pas encore stabilisées (tout le débat entourant les changements climatiques, par exemple).

Ces termes peuvent être :

- $\quad$ prédicatifs : amincissement de $X$ (amincissement de la couche d'ozone); X déverse Y dans $Z$ (les militaires déversent des déchets radioactifs dans la mer du Japon);

- $\quad$ quasi-prédicatifs (Polguère 2012) ${ }^{2}$ : aire de Y utilisée par X (aire de répartition de la la baleine grise);

- $\quad$ non prédicatifs : dioxyde de carbone, eau.

Nous nous intéressons ici plus particulièrement aux termes prédicatifs. Ces termes comprennent des verbes (boiser, planter, surexploiter), des noms (agriculture, monoculture, déboisement) et des adjectifs (durable, génétiquement modifie).

\subsection{Les limites des ressources terminologiques}

Les ressources terminologiques ne couvrent que partiellement les termes de nature prédicative. Si les nominalisations de verbes sont répertoriées, les verbes en contrepartie sont souvent omis (par exemple, recyclage, déboisement et déversement pourront être retenus, mais pas toujours recycler, déboiser ou déverser). Les adjectifs sont retenus lorsqu'ils apparaissent dans un syntagme nominal plus long (par exemple, une entrée pourra être consacrée à développement durable, mais pas à l'adjectif durable qui modifie pourtant d'autres noms, comme agriculture, exploitation, foresterie).

Par ailleurs, même si des termes prédicatifs sont intégrés à la nomenclature de ressources terminologiques, rien n'est fait pour les distinguer des autres termes et prendre en compte leur nature linguistique particulière (notamment, le fait qu'ils ont des arguments). Ils sont accompagnés d’une définition et, dans les thésaurus et ontologies, reliés à d'autres concepts au moyen de relations le plus souvent taxinomiques (hyperonymie, hyponymie). 


\subsection{Prise en compte des propriétés lexico-sémantiques des termes}

On retrouve dans la littérature terminologique un nombre croissant de propositions visant à intégrer les unités prédicatives dans les ressources terminologiques et à décrire leurs propriétés linguistiques (Lerat 2002 ; L’Homme 2012a ; Ingrosso et Polguère 2015, entre autres).

Ces travaux ont mis en évidence l'importance de prendre en compte les arguments dans la description des termes, mais également le fait que les unités de nature prédicative entretiennent avec d'autres termes un réseau très complexe de relations lexicales. Le tableau 1 (tiré de L’Homme 2012a) montre différentes propositions de représentation de la structure argumentale de verbes spécialisés.

Tableau 1 : Représentations diverses de la structure argumentale de verbes spécialisés (L’Homme 2012a)

\begin{tabular}{|c|c|c|}
\hline Verbe & Structure argumentale & Auteur \\
\hline contracter & Classes d'objets : HUMAIN contracter (v. tr.) $\mathrm{N}<$ obligation $>$ & Lerat (2002) \\
\hline contaminer & $\begin{array}{l}\text { Classes sémantiques : X (AGENT INFECTIEUX) } \sim \text { Y (ANIMAL; } \\
\text { PRÉPARATION; SUBSTANCE BIOLOGIQUE; SUBSTANCE COMPLEXE, } \\
\text { MATÉRIAU) }\end{array}$ & Tellier (2007) \\
\hline acquit $_{1}$ & Étiquettes d'éléments d'un cadre : Judge $\sim$ Defendant of Charges & Pimentel (2010) \\
\hline télécharger & $\begin{array}{l}\text { Rôles sémantiques : Agent } \sim \text { Patient de Source à Destination } \\
\text { Termes typiques : Utilisateur } \sim \text { fichier; logiciel de réseau, ordinateur à ordinateur }\end{array}$ & L’Homme (2010) \\
\hline
\end{tabular}

La figure 1 montre quant à elle le réseau terminologique construit à partir des deux acceptions du verbe éroder (DiCoEnviro visuel 2015).

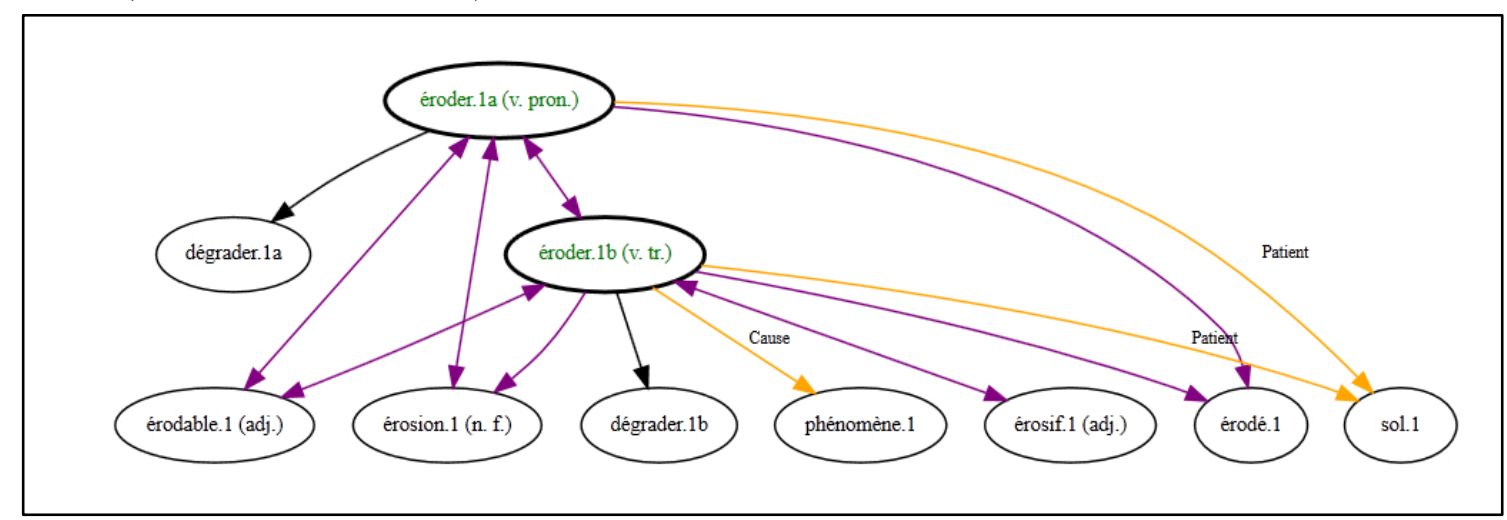

Figure 1 : Réseau terminologique des deux acceptions du verbe éroder (DiCoEnviro visuel 2015)

Même si ces travaux plus récents révèlent des aspects intéressants du lexique spécialisé, ils restent muets sur la contribution des unités prédicatives à la représentation des connaissances dans un domaine. La littérature propose, d'une part, des modèles de représentation des connaissances comme celles qui ont été évoquées en introduction qui disent peu de choses sur les propriétés linguistiques des termes et, d'autre part, des modèles descriptifs qui se focalisent sur les propriétés linguistiques des termes (ou une partie d'entre elles) et qui ne tentent pas de connecter ces descriptions à la connaisance spécialisée. La Sémantique des cadres (Fillmore 1976 ; Fillmore and Atkins 1982; Fillmore and Baker 2010) est une solution possible à ce problème. Elle pourrait à notre avis réconcilier les deux types de description.

\section{Sémantique des cadres et FrameNet}

La Sémantique des cadres (Fillmore 1976; Fillmore and Baker 2010) tient pour acquis que le lexique est construit sur la base de « connaissances d'arrière-plan » (de background knowledge). La structure de ces connaissances est représentée au moyen de «cadres » (en anglais, frames), définis comme des scénarios 
conceptuels qui fédèrent les réalisations dans le lexique. Par exemple, le cadre de la collecte de nourriture (Food_gathering) représente une situation dans laquelle interviennent des participants (en l'occurrence, GATHERER, CROP, SOURCE, comme participants obligatoires, ainsi que BENEFICIARY, CIRCUMSTANCES, DEGREE, etc., comme participants optionnels). Ce cadre regroupe des unités lexicales (UL) comme bring in.v, gather.v, harvest.v, pick.v, qui «évoquent » ce cadre (FrameNet 2015). La définition d'un cadre implique la découverte de participants appelés éléments du cadre (frame elements) et sont définis comme des rôles sémantiques propres (et uniques) à la situation décrite. Les participants non obligatoires sont appelés éléments de cadre centraux (core frame elements); ceux qui sont optionnels, éléments de cadres périphériques (non-core frame elements).

La ressource FrameNet (2015) décrit les cadres et leur réalisation sur le plan lexical. En plus de donner une description générale de nombreux cadres sémantiques, FrameNet propose des entrées consacrées aux unités lexicales évoquant ces cadres ainsi que des annotations contextuelles. Ces dernières permettent de voir l'ancrage entre la description abstraite d'un cadre (qui se veut une modélisation conceptuelle), les réalisations linguistiques de l'unité lexicale (définie comme cible de l'annotation) et ses interactions avec les éléments de cadre en corpus. Enfin, des relations sont établies entre cadres sémantiques.

Le modèle de la Sémantique des cadres a retenu l'attention d'un certain nombre de terminologues et de linguistes intéressés par le lexique d'un domaine de spécialité. On a proposé son application au domaine du football (Schmidt 2009 ; Dicionário da Copa do Mundo 2014), à la biomédecine (Dolbey et al. 2006 ; Wandji et al. (2013) ; au droit (Pimentel (2012) et à l'environnement (Faber 2012 ; ainsi que le travail décrit dans ces pages).

\section{Une « nouvelle » ressource terminologique}

Un domaine spécialisé comme celui de l'environnement comporte bien entendu des situations qui lui sont propres (différents types de changements, des conséquences de ces changements, des activités humaines ayant un impact sur l'environnement, des mesures prises pour contrer la dégradation, etc.). Nous tenons pour acquis que les termes évoquent des situations spécifiques au domaine dont ils relèvent et que des ensembles de termes peuvent évoquer la même situation. Les exemples suivants montrent des contextes dans lesquels des termes (en majuscules) dénotant des processus selon lesquels la composition des matières organiques subissent une transformation.

Les plastiques peuvent être BIODÉGRADABLES sans nécessairement être compostables

Traitement en milieu terrestre (par exemple, BIODÉGRADATION de déchets liquides ou de boues dans les sols, etc.).

... les arbres morts ont une fâcheuse tendance à SE DÉCOMPOSER

Un plastique biodégradable SE DÉGRADE dans l'environnement

Évaluer l'opportunité de mettre en place un système de compostage des matières PUTRESIBLES.

Outre le fait qu'ils aient grosso mode la même dénotation, ces termes ont en commun le fait d'avoir un seul argument ( $X$ se décompose ; $X$ est biodégradable). Cet argument dénote une entité qui subit un processus (c’est un Patient). Enfin, les réalisations de cet argument sont de même nature : il s’agit dans tous les cas de matières organiques.

Ces termes (après la réalisation d'étapes méthodologiques sur lesquelles nous revenons à la section 5) peuvent être réunis dans un cadre dont la description est reproduite à la figure 2.

\footnotetext{
Rotting

Definition: Example(s):

A Patient, which is an organic substance, undergoes the natural process of decaying.

These deadwood components DECOMPOSE rather quickly under the warm and humid conditions of tropical rain forests

Treating BIODEGRADABLE waste before it enters a landfill reduces global warming from fugitive
} 


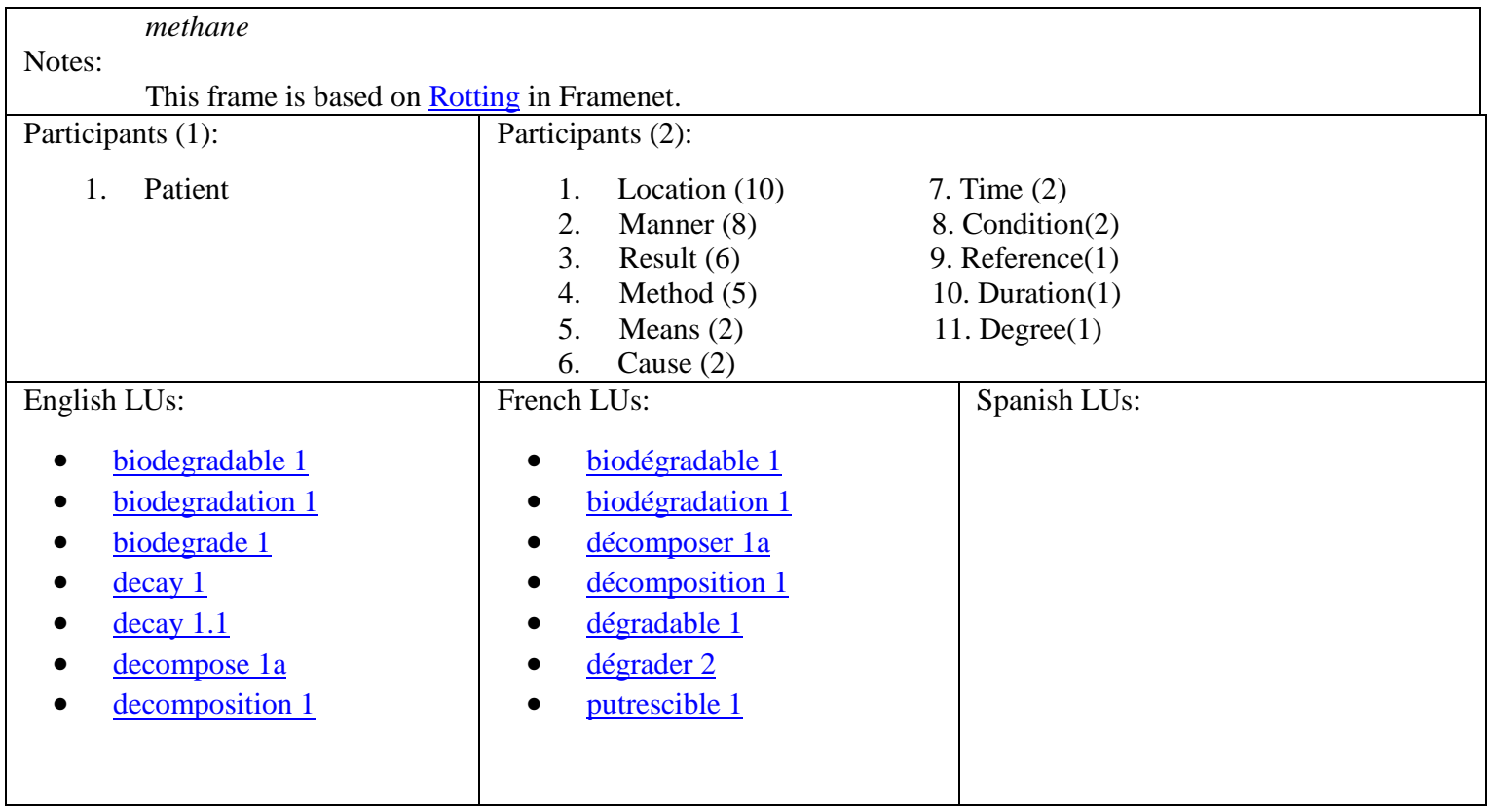

Figure 2 : Cadre Rotting (Framed DiCoEnviro 2016)

La description d'un cadre comporte une définition, quelques exemples anglais et une note pour indiquer si on s’inspire d'un cadre présent dans FrameNet. La seconde partie de la description énumère les participants obligatoires (éléments de cadre centraux) et optionnels (éléments de cadres périphériques). Enfin, trois listes de termes avec des liens vers des entrées lexicales sont données. On remarque que le cadre renferme des termes anglais et prévoit de l'espace pour des termes espagnols. Bien que cet article se focalise sur les termes français, le travail est également réalisé sur l’anglais et l'espagnol.

La figure 3 est une reproduction de l'entrée consacrée au terme biodégradable dans la ressource terminologique associée à la description des cadre, le DiCoEnviro (2016). Comme on peut le voir, chaque entrée donne la structure actancielle du terme, une liste de contextes, des liens lexicaux et, enfin, des équivalents dans d'autres langues ${ }^{3}$.

biodégradable $_{1}$, adj.

Domaine : gestion des matières résiduelles

biodégradable : matières $_{1}$ (;)

\section{Contextes}

\section{Contextes annotés}

- La quantité de biogaz produite à l'issue du procédé de biométhanisation est fonction des caractéristiques de l'effluent traité, en particulier de son contenu en matière organique biodégradable. (Source : BRODEUR)

- Selon les spécialistes en chimie, le nonylphénol est considéré comme un produit toxique difficilement

biodégradable qui altère le rythme reproductif des poissons. (Source : KABONGO)

- Les plastiques peuvent être biodégradables sans nécessairement être compostables. (Source : GERVAIS) Liens lexicaux

\begin{tabular}{|c|c|}
\hline Explication & Terme relié \\
\hline \multicolumn{2}{|r|}{ Voisins } \\
\hline$\approx$ & dégradable $_{1}$ \\
\hline$\approx$ & compostable $_{1}$, organique ${ }_{1}$, périssable ${ }_{1}$, putrescible 1 \\
\hline \multicolumn{2}{|c|}{ Autres parties du discours et dérivés } \\
\hline Verbe de sens voisin & biodégrader \\
\hline
\end{tabular}


Figure 3 : Entrée lexicale biodégradable 1 (DiCoEnviro 2016)

Enfin, un accès à des contextes annotés permet de voir de quelle manière le terme prédicatif se combine avec ses participants (obligatoires et optionnels). Une partie des contextes annotés de biodégradable $_{1}$ apparaissent à la figure 4 .

Outre les incidences néfastes pour l'environnement de ces lessives conventionnelles ${ }_{\text {[Patient] }}$ très peu [Degré BIODÉGRADABLES, combien de problèmes de santé sont en lien direct avec ces cocktails chimiques que nous appliquons chaque jour sur notre peau ? [PANACEA 127470 MCLH 24/08/2015]

Selon les spécialistes en chimie, le nonylphénol est considéré comme un produit toxique [Patient] difficilement $_{\text {[Manière] }}$ BIODÉGRADABLE qui altère le rythme reproductif des poissons. [KABONGO 0 MCLH 24/08/2015]

Elle permet de transformer la matière organique $_{[\text {Patient] }}$ facilement $_{[M a n i e ̀ r e]}$ BIODÉGRADABLE, qui est à l'origine des pollutions organiques et des odeurs. [PANACEA 173960 MCLH 24/08/2015] 


\begin{tabular}{|l|l|l|}
\hline \multicolumn{2}{|c|}{ Actants } & $\begin{array}{l}\text { lessive } \\
\text { matière } \\
\text { produit }\end{array}$ \\
\hline Patient & Tête (SN) (3) Autres \\
\hline \multicolumn{2}{|c|}{} \\
\hline Degré & Modificateur (SAdv) (1) & Peu \\
\hline Manière & Modificateur (SAdv) (2) & $\begin{array}{l}\text { difficillement } \\
\text { facilement }\end{array}$ \\
\hline
\end{tabular}

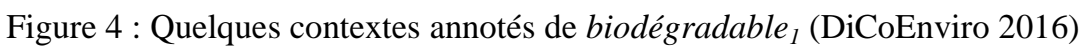

Les cadres sont également reliés entre eux selon différentes modalitées (héritage, causatif - inchoatif, précède, etc.; voir la section 5.2.3). Dans le Framed DiCoEnviro, le cadre Rotting hérite d'un cadre plus général appelé Undergo_change_of_state (qui renferme les verbes intransitifs changer, évoluer, fluctuer, modifier, varier ainsi que les noms changement, évolution, fluctuation, modification, variation).

Dans de nombreux cas, les cadres reliés fournissent une représentation de scénarios plus complexes rendant compte de la manière dont se déroulent les événements dans le domaine de l'environnement. La figure 5 donne un aperçu des cadres associés aux activités humaines, notamment celles qui ont un impact sur l'environnement ainsi que les termes évoquant quelques-uns de ces cadres.

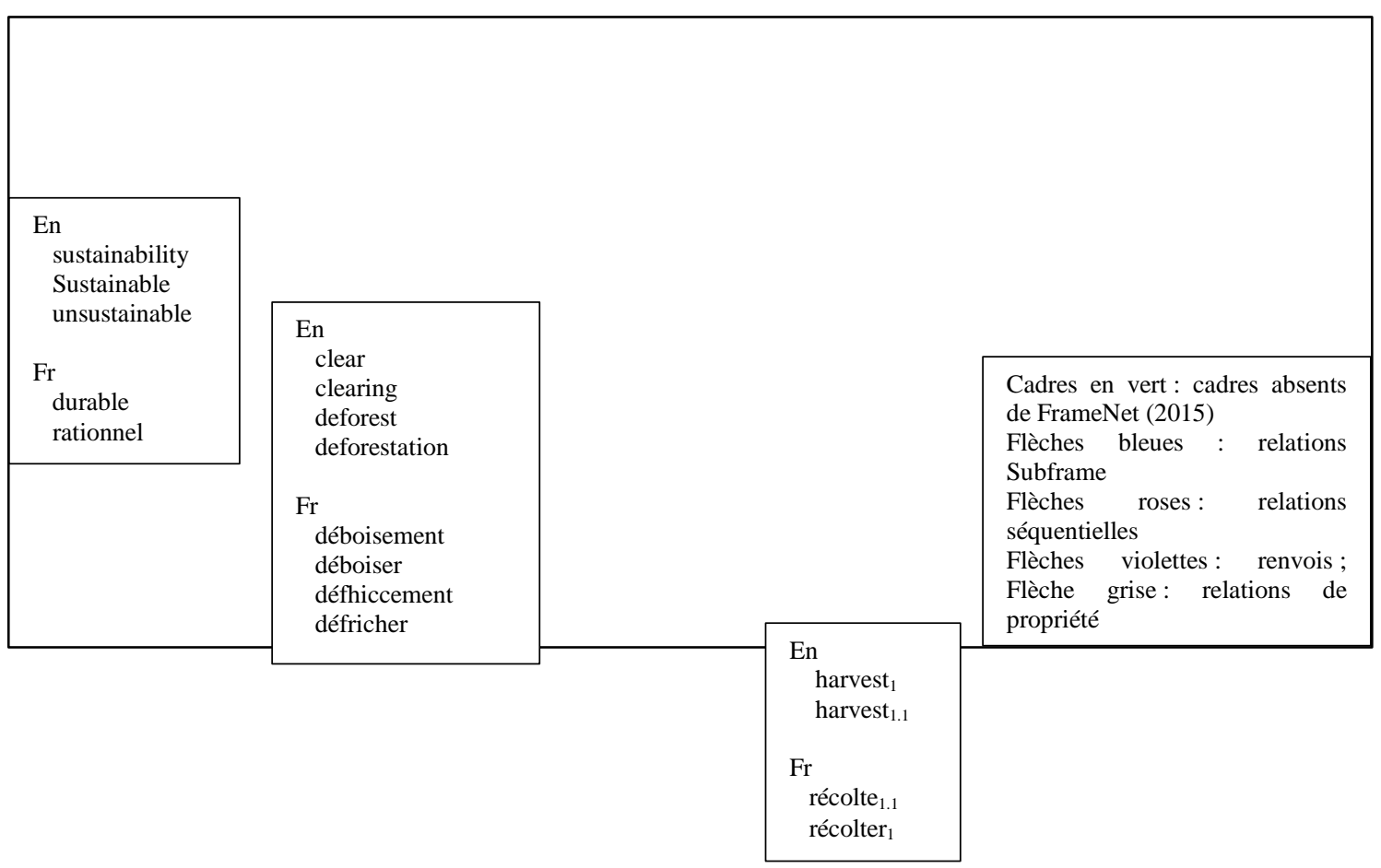

Figure 5 : Cadres associés aux activités humaines (Framed DiCoEnviro 2016)

\section{Considérations méthodologiques}

La méthodologie mise au point pour la description des termes, des cadres et l'établissement des relations entre cadres s’inspire de celle développée dans le projet FrameNet (Fillmore et al. 2003 ; Ruppenhofer et al. 2010). Toutefois, quelques adaptations ont été faites afin de tenir compte du contexte terminologique dans lequel nous nous plaçons. Comme on le verra dans ce qui suit, notre méthode est essentiellement ascendante, puisqu'elle procède d'un corpus spécialisé et des termes qu’il renferme. Le terminologue, ne 
disposant pas a priori des connaissances du domaine ne peut pas s'appuyer avec une confiance absolue sur son intuition et s’inspirera plus directement des énoncés contextuels. En outre, il est extrêmement difficile, voire impossible, pour un non-expert, de dresser une première liste de termes pouvant évoquer un cadre, puisqu'il ne les connait pas a priori. Par exemple, si on examine le cadre Rotting donné à la figure 2, il est difficile pour un non-expert de savoir que le verbe pourrir, pourtant un candidat évident, s'utilise très peu dans les textes d'environnement.

Dans ce qui suit, nous ne retenons que les étapes méthodologiques nécessaires à la découverte et à définition de cadres sémantiques. Comme nous l'avons vu, la ressource lexicale renferme des rubriques (les liens lexicaux, par exemple), qui ne concernent pas directement les cadres sémantiques. Cette partie de la méthodologie se décline donc en sept étapes qui font l'objet des sections suivantes ${ }^{4}$. Les quatre premières sont mises en œuvre pour créer les entrées lexicales et les annotations : 1. Élaboration d'un corpus et sélection des termes; 2. Sélection de contextes; 3. Définition des structures actancielles; 4. Annotation des contextes. Les trois suivantes concernent plus directement la définition des cadres sémantiques et l'éblissement des relations entre ces cadres : 5 . Découvertes de cadres sémantiques; 6 . Définition des cadres sémantiques ; 7. Établissement de relations entre cadres. Nous nous attarderons davantage sur ces dernières. Les descriptions sont faites dans différentes fiches XML gérées par des schémas. Ces fiches sont interreliées au moment de l'affichage des données dans une interface Web.

\subsection{Entrées lexicales}

\subsubsection{Corpus et sélection de termes}

Tout le travail terminologique réalisé dans le contexte du projet dont nous traçons les grandes lignes ici s’appuie sur des corpus spécialisés regroupant des textes portant sur le domaine de l'environnement. Nous utilisons deux sortes de corpus. Dans un premier temps, nous assemblons des corpus manuellement. Comme le domaine de l'environnement est vaste et regroupe des thématiques fort différentes, nous avons pris la décision de distinguer les corpus par sous-domaines et de procéder à une sélection des termes un sous-domaine à la fois. Pour l'instant, nous disposons de corpus associés aux sous-domaines des changements climatiques, des énergies renouvelables, des espèces menacées, de l'électrification des transports, de la gestion des matières résiduelles et du déboisement. Les corpus ont des tailles variant entre 500000 et 1200000 mots. Nous utilisons également un corpus assemblé automatiquement dans le cadre du projet PANACEA ${ }^{5}$. Ce dernier est utilisé pour la recherche de contextes complémentaires puisque les textes qu'il renferme sont extrêmement variés.

Chacun des corpus est soumis à un extracteur de termes (TermoStat, Drouin 2003) qui propose une liste de candidats (noms, verbes, adjectifs et adverbes). La liste est ensuite examinée par un terminologue afin de retenir les termes valides.

En plus de statuer sur la nature terminologique d'un candidat, le terminologue pourra être appelé à distinguer les sens de formes ambiguës puisque l'extracteur, bien qu'il puisse désambiguïser des formes semblables appartenant à des parties du discours différentes, ne peut distinguer les sens. Par exemple, la forme récolte donnera lieu à deux entrées distinctes (périodes de récolte): 1. une activité qui consiste à recueillir des céréales ou autres plantes ; 2. Le résultat de cette activité (la perte de récoltes).

\subsubsection{Collecte de contextes}

Une fois établi le statut terminologique d'une unité lexicale, le terminologue procède à la collecte de contextes (jusqu'à 20) dans lesquels elle apparaît. Les contextes sont extraits au moyen d'un concordancier maison et choisis en fonction des critères comme leur provenance (sources variées) ; variété d'emplois d'un terme ; variété de réalisations d'actants, etc. Les contextes sont placés dans une fiche de format XML (qui renferme l'ensemble des renseignements sur les termes) accompagnés d'une abréviation qui renvoie à la source. Ces contextes sont ensuite annotés selon un protocole décrit à la section 5.1.4. 


\subsubsection{Description de la structure actancielle}

L'étape suivante consiste à décrire la structure actancielle des termes choisis. En fait, il s'agit d'abord d'une hypothèse que formule le rédacteur, hypothèse devant être confirmée au cours de la rédaction des autres rubriques servant à décrire le terme (lors de l'annotation des contextes, notamment).

En s'aidant des contextes choisis (section 5.1.2), le rédacteur définit d'abord le nombre de participants obligatoires (arguments ou actants); une correspondance partielle peut être établie avec « éléments de cadre centraux »). Pour récolter, par exemple trois actants ( $X$ récolte $Y$ de $Z$ ) sont identifiés. Les actants sont ensuite étiquetés pour indiquer leur rôle sémantique. En outre, un terme typique censé représenter l'unité attendue dans une position argumentale est donné.

$$
\begin{aligned}
& \text { récolter }_{1}, \mathrm{n} . \mathrm{f} \text {. } \\
& \text { AGENT }\{\text { homme } \sim \text { PATIENT\{végétal\} de SOURCE\{terre] }
\end{aligned}
$$

Ici, il convient de souligner que les étiquettes se distinguent de celles utilisées dans FrameNet. Nous avons déjà vu que le cadre Food_gathering fait appel à des étiquettes comme GATHERER, CROP et SOURCE. Les étiquettes utilisées dans FrameNet pour distinguer les éléments du cadre sont définies en fonction d'un cadre spécifique. Nous avons opté pour des étiquettes plus classiques pouvant s'appliquer à un grand nombre de termes.

\subsubsection{Annotation des contextes}

L'étape suivante consiste à annoter les contextes en tenant compte de la structure actancielle définie à la section 5.1.3. L'annotation est réalisée dans la fiche XML et indique les éléments suivants (une illustration de contextes annotés est donnée à la figure 4) :

- $\quad$ Le terme prédicatif;

- $\quad$ Les participants et leur nature (obligatoires ou optionnels);

- $\quad$ Le rôle sémantique des participants (ex. Agent, Patient, Source)

- $\quad$ La fonction syntaxique des participants (ex. complément, tête, modificateur);

- $\quad$ Le groupe syntaxique des participants (ex. SA, SP, SN);

- De l'information administrative (Source, statut de l'annotation, le code de l'annotateur, la date de la dernière mise à jour).

Comme on peut le voir, les annotations tiennent également compte des participants optionnels. L'annotation permet de montrer les réalisations possibles des participants dans des phrases extraites de corpus et de valider la structure actancielle définie préalablement.

\subsection{Cadres sémantiques}

\subsubsection{Découverte de cadres sémantiques}

La description des structures actancielles dans le DiCoEnviro ainsi que les annotations permettent de lancer un travail de découverte de cadres sémantiques. La méthodologie mise en œuvre dans le projet FrameNet (Fillmore et al. 2003) prévoit une première définition d'un cadre à partir de l'intuition des lexicographes. Celle-ci est ensuite validée et enrichie par des analyses en corpus. Dans le cas qui nous préoccupe, nous avons d'abord eu recours à une analyse empirique visant à faire émerger des régularités dans les données dont nous disposons (les entrées lexicales et les annotations). Ces régularités sont proposées au terminologue afin de l'assister dans l'analyse qu'il fera des termes. Cette analyse fait appel à deux méthodes ${ }^{6}$. 
La première méthode ${ }^{7}$ consiste à extraire les termes de la ressource lexicale et des annotations et à effectuer différents regroupements faisant ressortir des similitudes quant aux participants associés à ces termes. Le tableau 2 montre quels sont les renseignements extraits. Des tris peuvent être faits sur chacune de colonne avant de voir les éléments qu’ils peuvent possiblement partager.

Tableau 2 : Données extraites de la ressource lexicale et des annotations.

\begin{tabular}{|l|l|l|l|l|l|}
\hline & \multicolumn{1}{|c|}{ Structure actancielle } & Role 1 & Rôle 2 & Rôle 3 & \multicolumn{1}{c|}{ Autres participants } \\
\hline v. tr. & aggraver $_{1 \mathrm{~b}}:$ Cause $~$ Patient & Cause & Patient & -- & Degré, Fréquence, Lieu. Temps \\
\hline v. tr. & amplifier $_{1}$ : Cause $\sim$ Patient & Cause & Patient & -- & degré, Fréquence, Lieu. Temps \\
\hline v. tr. & augmenter $_{1 \mathrm{~b}}:$ Cause $~$ Patient & Cause & Patient & -- & Lieu, Manière, Résultat, Temps \\
\hline
\end{tabular}

La seconde méthode consiste évidemment à consulter FrameNet afin de repérer des cadres qui pourraient être appropriés aux sens des termes de l'environnement ${ }^{8}$.

\subsubsection{Définition des cadres sémantiques}

La définition des cadres repose principalement sur les critères donnés dans FrameNet (Ruppenhofer et al. 2010). Nous donnons ci-dessous ceux qui sont le plus souvent utilisés pour décrire les données de l'environnement ${ }^{9}$. À noter que nous avons adapté ci-dessous la terminologie donnée dans Ruppenhofer et al. (2010) (terme vs unité lexicale ; participant vs élément du cadre).

1. Tous les termes évoquant un cadre doivent avoir le même nombre d'arguments ${ }^{10}$. En outre, ces arguments doivent être de même type sémantique (appartenir aux mêmes classes).

Par exemple, les verbes défricher et déboiser ont respectivement trois actants ( $X$ défriche $Y$ de $Z$; $X$ déboise $Y$ de $Z$ ). En outre, dans les deux cas, le premier actant est réalisé par un actant de type humain (agriculteur; entreprise), le second, par un lieu (terre, région) et le troisième par des types d'arbres.

Les différences qui ne tiennent qu’à la réalisation syntaxique des actants n’est pas prise en compte (emplois passif vs actif, différences liées à l'aspect ou au temps, etc.).

2. La dénotation fondamentale des termes d'un cadre sémantique doit être semblable.

Ce critère a permis de distinguer les trois cadres Change_position_on_a_scale (s’inspirant de FrameNet), Change_of_impact et Change_of_feature (ces deux derniers ont été créés pour les données d'environnement). Cet exemple permet également de constater que certains cadres ont été adaptés à notre contexte spécialisé. Le premier cadre renferme des unités lexicales dénotant un changement que subit un participant quant à son importance numérique ou son étendue (augmentation, baisse, diminution, s'élever). Le second cadre structure une situation dans laquelle un participant change, mais cette fois le changement est associé à une manière, à savoir, une plus grande fréquence ou une gravité (accélération, s'agraver, s'intensitifer, ralentir). Le dernier cadre décrit une situation dans laquelle une entité subit un changement qui l'amène à changer de forme (amincissement, s'éroder, reculer, retrait).

3. Les presuppositions et les attentes relatives aux termes d'un cadre doivent être partagées.

Par exemple, de nombreux termes dénotent des méthodes utilisées par les experts pour mieux comprendre les changements climatiques: calculer, estimer, modéliser, simuler. Calculer engage trois participants : un expert, un élément faisant l'objet du calcul et une méthode. Estimer engage les mêmes actants, mais ici une valeur est attendue. Enfin, modéliser et simuler engagent un expert, un élément à imiter et une reproduction de cet élément. Ainsi, calculer, estimer évoquent deux cadres sémantiques distincts; modéliser et simuler évoquent tous les deux un troisième cadre. 
4. Les actants d'un terme qui sont mis en évidence et ceux qui sont placés en arrière-plan doivent être les mêmes à l'intérieur d'un cadre.

En vertu de ce critère, les termes se déplacer (un Québécois qui se déplace en transport collectif (train, avion, autobus) et transporter (Chaque navette peut transporter de 60 à 75 passagers assis confortablement) évoquent des cadres distincts. Se déplacer met en évidence le passager et place le moyen de transport en arrière-plan; transporter met plutôt en évidence le moyen de transport et place le passager en arrière-plan.

5. Les antonymes de type complémentaires (sustainable, unsustainable), gradables (chaud, froid) et certains types de réversifs (se réchauffer, se refroidir) sont regroupés dans le même cadre. Toutefois, les conversifs, comme ils mettent en évidence des éléments de cadre différents (voir critère 4) et d'autres types de réversifs (comme déboiser, reboiser qui font appel à des actants de rôles différents : une source ou une destination) sont placés dans des cadres différents.

Évidemment, un item lexical peut évoquer plus d'un cadre et sera par conséquent répertorié à des endroits différents. C'est le cas de l'item dégrader qui peut évoquer les cadres Rotting (une matière se dégrade), Damaging (certaines émissions humaines (les CFC) dégradent l'ozone stratosphérique) et Deteriorating (la qualité de l'eau se dégrade).

Nous avons déjà mentionné (section 5.2.1) que la ressource FrameNet est consultée pour tenter d'établir des correspondances avec nos données. Bien entendu, certains termes évoquent des cadres qui n’ont pas encore été décrits dans FrameNet. Par exemple, tous les adjectifs évoquant la propriété de n’être pas dommageable pour l'environnement (écologique 2 , environnemental 2 , propre , vert $_{1}$ ). Enfin, certaines situations, même si elles sont décrites dans FrameNet font appel à un nombre de participants différents.

Les cadres sont définis dans un fichier XML. La figure 2 montre un exemple de cadre défini tel qu’il apparaît dans le Framed DiCoEnviro. Rappelons que les différences observées avec FrameNet sont mentionnées dans la rubrique « Note » apparaissant dans la description du cadre.

\subsection{3 Établissement de relations entre cadres sémantiques}

Le travail de définition des cadres a rapidement fait ressortir l’intérêt de rendre compte des liens partagés par certains d'entre eux : par exemple, de nombreux cadres décrivent des sortes de changements (qui surviennent ou qui sont causés par quelque chose), des activités humaines, des méthodes utilisées pour comprendre les changements climatiques, etc. Les relations entre cadres, comme nous avons eu l'occasion de le souligner, permettent de décrire des scénarios plus complexes rendant compte d'événements qui se produisent dans le domaine.

Nous avons d'abord fait appel à la liste de relations décrites dans FrameNet. Celle-ci compte huit jeux de relations bidirectionnelles (les exemples sont les nôtres et non ceux de FrameNet; une partie de ces relations sont illustrées graphiquement à la figure 5) :

- Héritage : Inherits, Is inherited by (ex. Rotting inherits Undergo_change_of_state);

- Perspective : Perspective on, Is perspectivized in (ex. Accumulating Perspectives Greenhouse_effet);

- Utilisation : Uses, Is used by (ex. Predicting_scenario Uses Representation);

- $\quad$ Sous-cadre : Subframe of, Has subframe (ex. Planting Subframe of Agriculture);

- Chronologie : Precedes; Is preceded by (ex. Planting Precedes Food_gathering);

- Causation : Is inchoative of; Is causative of (ex. Change_position_on_a_scale Is inchoative of Cause_change_of_position_on_a_scale);

- Voir aussi : See also (ex. Adding_trees_in_location See also Planting). 
Nous avons également ajouté deux relations que nous estimons valables pour rendre compte des cadres identifiés à partir des termes d'environnement. Il s'agit de :

- Opposition : Is opposed to (ex. Removing_trees_from_location Is opposed to Adding_trees_in_location). Nous avons ajouté cette relation pour rendre compte de liens entre cadres évoquant des situations inverses (Removing_trees_from_location renferme les termes déboisement, déboiser, défrichement, défricher; Adding_trees_in_location, les termes boisement, boiser, reboisement, reboiser).

- Propriété : Is a property of, Has property (ex. Sustainability is a property of Human_activity). Cette relation permet de rendre compte du lien entre des propriétés fortement associées à une situation décrite par un autre cadre. Sustainability renferme le terme durable; Human_activity, le terme activité; par ailleurs, un vaste ensemble d'activités humaines sont décrites dans des sous-cadres de Human_activity (voir figure 5).

\section{Conclusion}

Nous avons proposé un nouveau type de ressource terminologique qui tient compte de termes de nature prédicatives dans le domaine de l'environnement et qui décrit leurs propriétés lexico-sémantiques. Un module lexical décrivant notamment leur structure actancielle est couplé à un module d'annotations qui montre de quelle manière le terme prédicatif se combine avec ses participants dans des phrases extraites de corpus spécialisés.

Par ailleurs, nous avons montré que les entrées lexicales peuvent ensuite être reliées à une forme de représentation conceptuelle. Cette représentation, qui s’inspire de la Sémantique des cadres, réunit les termes en fonction des situations qu'ils évoquent en tenant pour acquis que des termes évoquant une même situation partagent forcément des traits lexico-sémantiques. De plus, les cadres reliés mettent au jour des scénarios plus complexes montrant les interrelations entre événements se produisant dans le domaine. L’intérêt de la Sémantique des cadres et de son adaptation à un contexte terminologique résident dans le fait que la représentation conceptuelle ne fait plus l'impasse sur les propriétés lexicosémantiques des termes. Au contraire, ce sont ces propriétés lexico-sémantiques qui guident sa définition.

Enfin, nous avons défini une méthodologie afin de recueillir les termes et les contextes dans lesquels ils apparaissent, de les décrire dans des entrées lexicales et des annotations. Nous avons également montré de quelle manière les cadres sont découverts et définis. Nous avons jusqu'à maintenant défini 125 cadres (dont plus de la moitié ont été créés pour des termes d’environnement). Ces cadres renferment 395 termes français dont la plupart sont prédicatifs.

Le travail se poursuit (essentiellement en anglais et en français) et nous espérons pouvoir ajouter des termes quasi-prédicatifs à nos descriptions. Nous explorons également différentes méthodes automatiques afin de systématiser et d’accélérer le travail descriptif.

\section{Remerciements}

Le projet décrit dans les pages qui précèdent s’inscrit dans un projet soutenu financièrement par le Conseil de recherches en sciences humaines (CRSH) du Canada. J'aimerais remercier deux relecteurs anonymes dont les commentaires ont permis de clarifier certains aspects abordés dans l'article.

\section{Références bibliographiques}

Bernier-Colborne, G. \& M.C. L’Homme (2015). Using a distributional neighbourhood graph to enrich semantic frames in the field of the environment. In Actes de la 10e conférence internationale Terminology and Artificial Intelligence (TIA'15), Granada, Spain, 9-16. 
DiCoEnviro. Dictionnaire fundamental de l'environnement (http://olst.ling.umontreal.ca/cgibin/dicoenviro/search.cgi). Page consultée le 10 mars 2016.

Dolbey, A., M. Ellsworh \& J. Scheffczyk (2006). BioFrameNet: A Domain-specific FrameNet Extension with Links to Biomedical Ontologies. KR-MED 2006 Biomedical Ontology in Action, Baltimore, Maryland.

Drouin, P. (2003). Term Extraction Using Non-Technical Corpora as a Point of Leverage. Terminology 9(1), 99-117.

EIONET, GEMET Thesaurus (http://www.eionet.europa.eu/gemet/). Page consultée le 23 novembre 2015.

Envo. Environmental Ontology (http://environmentontology.org/). Page consultée le 23 novembre 2015.

Faber, P. (ed.) (2012). A Cognitive Linguistics View of Terminology and Specialized Language. Berlin/New York: Mouton de Gruyter.

Fillmore, C.J. (1976). Frame Semantics and the Nature of Language. In Annals New York Academy of Sciences: Conference on the Origin and Development of Language and Speech 280, 20-32.

Fillmore, C.J., \& B.T. Akins (1992). Toward a Frame-based Lexicon: The Semantics of RISK and its Neighbors. In A. Lehrer and E. Kittay (eds.) Frames, Fields, and Contrast: New Essays in Semantics and Lexical Organization. Hillsdale: Lawrence Erlbaum Associates, 75-102.

Fillmore, C.J. \& Collin Baker (2010). A frames approach to semantic analysis. In The Oxford Handbook of Linguistic Analysis, Bernd Heine and Haiko Narrog (eds), Oxford : Oxford University Press, 313-339.

Fillmore, C., M.R.L. Petruck, J. Roppenhofer \& A. Wright (2003). FrameNet in Action: The Case of Attaching. International Journal of Lexicography 16(2), 297-332.

Framed DiCoEnviro. A Framed Version of DiCoEnviro (http://olst.ling.umontreal.ca/dicoenviro/framed/index.php). Page consultée le 10 mars 2016.

FrameNet (https://framenet.icsi.berkeley.edu/fndrupal/home). Page consultée le 23 novembre 2015.

IATE, Interactive Terminology for Europe (http://iate.europa.eu/SearchByQueryLoad.do?method=load). Page consultée le 23 novembre 2015.

Ingrosso, F. \& A. Polguère (2015). How Terms Meet in Small-World Lexical Networks: The Case of Chemistry Terminology. Proceedings of the Conference Terminology and Artificial Intelligence 2015, 167-171.

L’Homme, M.C. (2012a). Le verbe terminologique : un portrait de travaux récents. In Neveu, F. et al. (éd.). Actes du 3e Congrès mondial de linguistique française, Lyon, France, EDP Sciences.

L'Homme, M.C. (2012b). Using ECL (Explanatory Combinatorial Lexicology) to discover the lexical structure of specialized subject fields, In Apresjan, J. et al. (eds.). Words, Meanings and other Interesting Things. A Festschrift $n$ Honour of the 80th Anniversary of Professor Igor Alexandrovic Mel'cuk, Moscow: RCK, 378-390.

L’Homme, M.C. (2015). Découverte de cadres sémantiques dans le domaine de l'environnement: le cas de l'influence objective. Terminàlia 12, 29-40.

L’Homme, M.C., B. Robichaud \& C. Subirats (2014). Discovering frames in specialized domains, In Language Resources and Evaluation, LREC 2014, Reykjavik, Iceland.

Lerat, P. (2002). Qu’est-ce que le verbe spécialisé ? Le cas du droit. Cahiers de Lexicologie 80, 201-211.

Mel'čuk. I., A. Clas et A. Polguère (1995). Introduction à la lexicologie explicative et comibinatoire, Louvain-laNeuve : Duculot.

Pimentel, J. (2010). Description de verbes juridiques au moyen de la sémantique des cadres, Terminologie \& Ontologie : Théories et applications (Toth 2011), Annecy 2011, 26-27 mai 2011.

Pimentel, J. (2013). Methodological Bases for Assigning Terminological equivalents. A Contribution. Terminology 19(2), 237-257.

Polguère, A. (2012). Propriétés sémantiques et combinatoires des quasi-prédicats sémantiques. Scolia 26, 131-152.

Ruppenhofer, J, M. Ellsworth, M. Petruck, C. Johnson \& J. Scheffczyk (2010). FrameNet II: Extended Theory and Practice (http://framenet.icsi.berkeley.edu). Page consultée le 30 novembre 2014. 
Schmidt, T. (2009). The Kicktionary - A Multilingual Lexical Resources of Football Language. In Boas, H.C. (ed). Multilingual FrameNets in Computational Lexicography. Methods and Applications, Berlin/NewYork: Mouton de Gruyter, 101-134.

Tellier, C. (2008). Verbes spécialisés en corpus médical : une méthode de description pour la rédaction d'articles terminographiques, Travail dirigé présenté au Département de linguistique et de traduction, Université de Montréal.

Wandji, O., M.C. L’Homme and N. Grabar (2013). Discovery of Semantic Frames for a Contrastive Study of Verbs in Medical Corpora. Terminology and Artificial Intelligence, TIA 2013, Paris.

${ }^{1}$ Par ailleurs, un nombre croissant d'applications faisant appel au traitement automatique des langues (TAL) exploitent les textes spécialisés et exigent une caractérisation fine des propriétés linguistiques des termes qu’ils renferment.

${ }^{2}$ Les termes quasi-prédicatifs (ou, de manière plus générale, les unités lexicales quasi-prédicatives) sont des noms qui dénotent des entités (contrairement aux termes prédicatifs qui dénotent des événements ou des caractéristiques). Il s'agit de termes qui ont un sens liant, c'est-à-dire un sens qui fait appel à des participants (d'après Polguère 2012). La nature quasi-prédicative du terme peut être démontrée par ses propriétés de combinatoire : a) un participant peut se réaliser sous la forme d'un dépendant (par exemple, un complément) du terme quasi-prédicatif (par exemple aire de répartition) ; b) un participant se manifeste également dans les collocations contrôlées par le terme quasi-prédicatif (un X (une espèce) peuple l'aire ; l'aire abrite X (l'espèce)).

${ }^{3}$ Le modèle d'entrée lexicale s'appuie sur la Lexicologie explicative et combinatoire, LEC (Mel'čuk et al. 1995). L'intérêt de la LEC pour la terminologie a été souligné par un certain nombre d'auteurs. Nous avons montré (L’Homme 2012b) de quelle manière le modèle appliqué à des termes d'environnement permet de dégager la structure lexicale du domaine par le repérage et l'encodage systématique de liens paradigmatiques et syntagmatiques. Dans l'application que nous en faisons, le module de description des cadres regroupe des entrées lexicales et permet de fournir une vue complémentaire à celle fournie par la LEC. Cette vue, à notre avis, est celle qui reflèterait le lien entre les termes et les connaissances du domaine.

${ }^{4}$ La méthodologie est illustrée au moyen d’un cadre spécifique, celui de l’influence objective, dans L’Homme (2015).

${ }^{5}$ Le corpus PANACEA est disttribué gratuitement à des fins de recherche à l'adresse http://catalog.elra.info/product_info.php?products_id=1184.

${ }^{6}$ Nous explorons actuellement des méthodes de découverte automatisée du lexique associée à des cadres à partir de grands corpus, méthode s'appuyant sur l'analyse distributionnelle (Bernier-Colborne et L’Homme 2015).

${ }^{7}$ Le détail des deux méthodes et donné dans L’Homme et al. (2014).

${ }^{8}$ Cette comparaison est partiellement automatisée pour les termes anglais : les données de notre ressource lexicale sont comparées à celle de FrameNet afin de localiser plus rapidement les correspondances possibles.

${ }^{9}$ Parmi les autres critères décrits dans Ruppenhoffer et al. (2010), on trouve : 1. dans les cadres qui sont complexes sur le plan aspectuel, les termes doivent impliquer le même ensemble d'étapes et de transitions ; 2 . les éléments de cadre doivent entretenir le même type d'interrelations au sein d'un cadre sémantique; 3. les différences de registre (niveau de langue, variation géographique, etc.) ne donnent pas lieu à des cadres différents.

${ }^{10}$ Les cadres peuvent toutefois contenir des termes se distinguant en fonction de l'alternance de leurs actants. Par

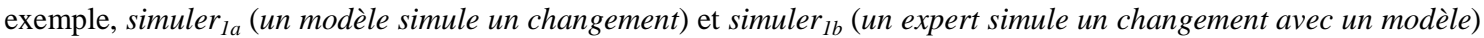
sont placés dans le même cadre. La ressource lexicale distingue ces acceptions, mais nous estimons que les deux termes évoquent le même cadre sémantique. 